Supporting Information for:

\title{
Realizing High Figure of Merit in Phase-Separated
}

\section{Polycrystalline $\mathrm{Sn}_{1-x} \mathrm{~Pb} \mathrm{~b}_{x} \mathrm{Se}$}

Guodong Tang,,${ }^{1,}$ Wei Wei, ${ }^{1}$ Jian Zhang, ${ }^{2,}{ }^{*}$ Yusheng Li, ${ }^{1}$ Xiang Wang, ${ }^{1}$ Guizhou Xu, ${ }^{1}$

Cheng Chang, ${ }^{4}$ Zhihe Wang, ${ }^{3}$ Youwei Du, ${ }^{3}$ Li-Dong Zhao ${ }^{4}{ }^{*}$

${ }^{1}$ School of Materials Science and Engineering, Nanjing University of Science and Technology, Nanjing 210094, China.

${ }^{2}$ Key Laboratory of Materials Physics, Institute of Solid State Physics, Chinese Academy of Sciences, Hefei 230031, China.

${ }^{3}$ National Laboratory of Solid State Microstructures, Department of Physics, Nanjing University, Nanjing 210093, China.

${ }^{4}$ School of Materials Science and Engineering, Beihang University, Beijing 10091, China.

Corresponding authors:

*tangguodong@,njust.edu.cn (G.T.); zhangjian@issp.ac.cn (J. Z.);

zhaolidong@buaa.edu.cn (L.-D.Z.).

\section{Content:}

Table S1

Figure S1

Figure S2

Figure S3

Figure S4

Figure S5 
Table S1: The lattice parameters extracted from cell refinement for $\mathrm{SnSe}+x \% \mathrm{PbSe}$ samples. Data of $\operatorname{SnSe}(x=0)$ from standard JCPDS card (No. 48-1224) is shown for comparison.

\begin{tabular}{|l|l|l|l|}
\hline SnSe & $\mathrm{a}(\mathrm{A})$ & $\mathrm{b}(\mathrm{A})$ & $\mathrm{c}(\AA)$ \\
\hline$X=0$ & $11.49(7)$ & $4.15(3)$ & $4.44(0)$ \\
\hline$X=1$ & $11.49(5)$ & $4.15(4)$ & $4.43(6)$ \\
\hline$X=2$ & $11.49(1)$ & $4.15(5)$ & $4.43(6)$ \\
\hline$X=3$ & $11.49(1)$ & $4.15(1)$ & $4.43(1)$ \\
\hline
\end{tabular}


Figure S1. Temperature dependence of specific heat for bulk polycrystalline SnSe + $\mathrm{x} \% \mathrm{PbSe}$ samples.

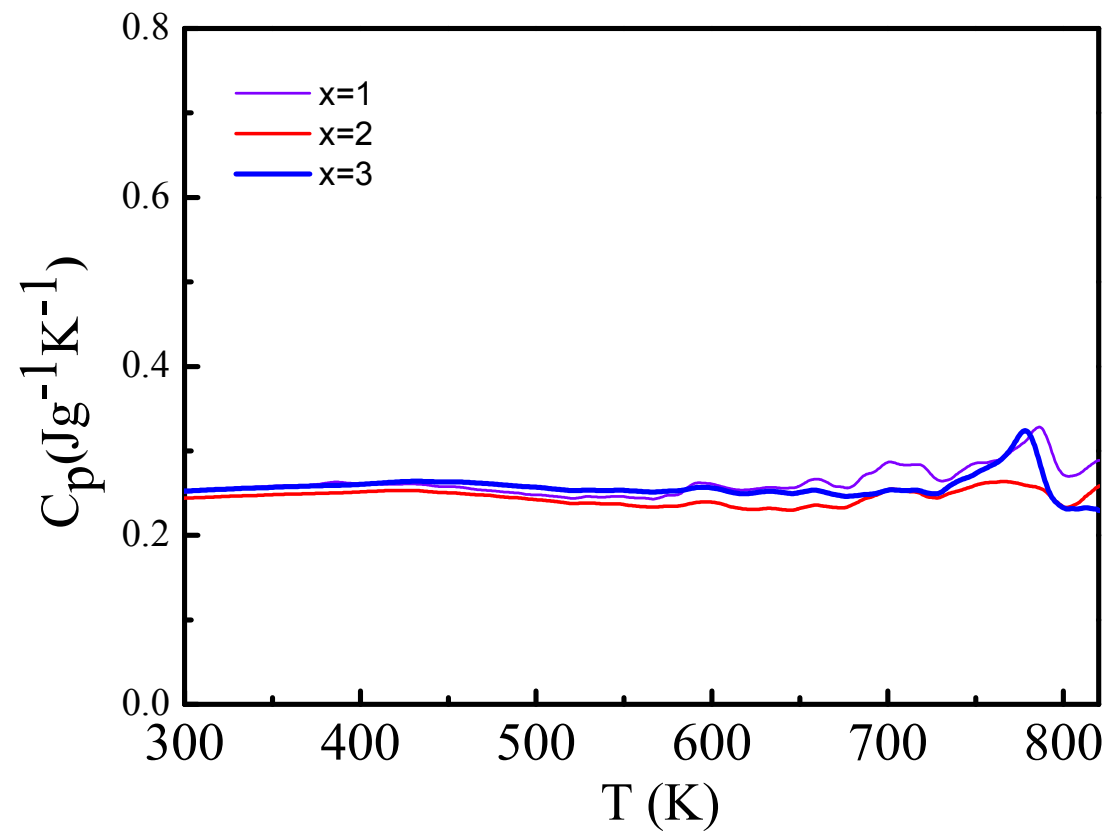


Figure S2. Measured Hall carrier concentration as a function of PbSe content at room temperature.

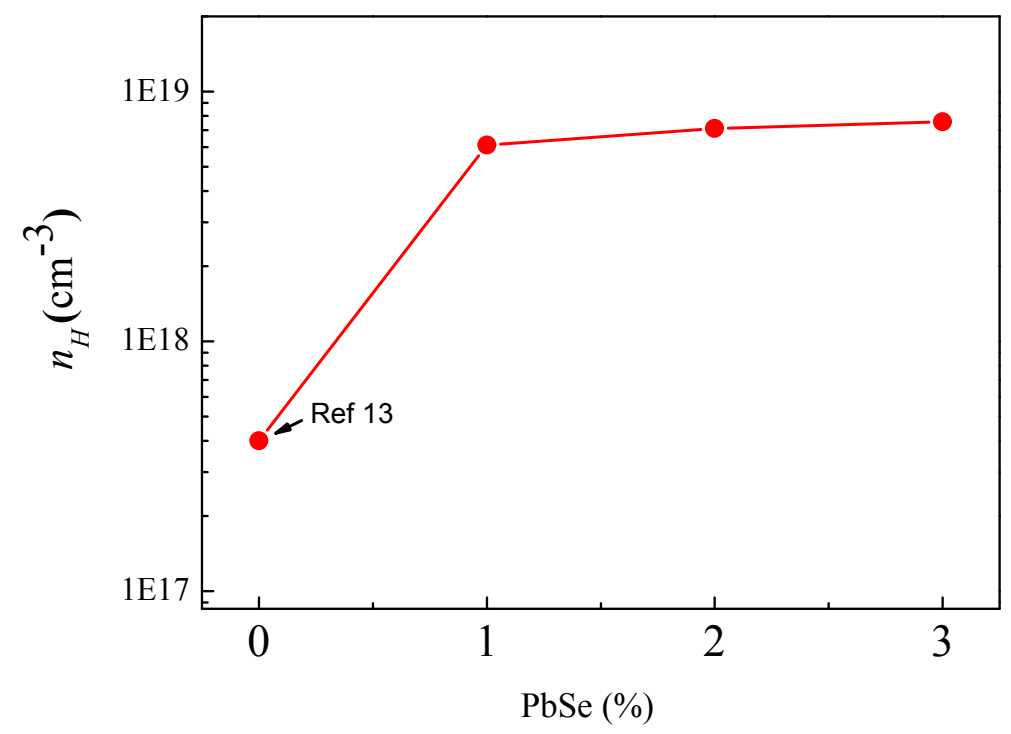


Figure S3. Carrier mobility as a function of $\mathrm{PbSe}$ content at room temperature.

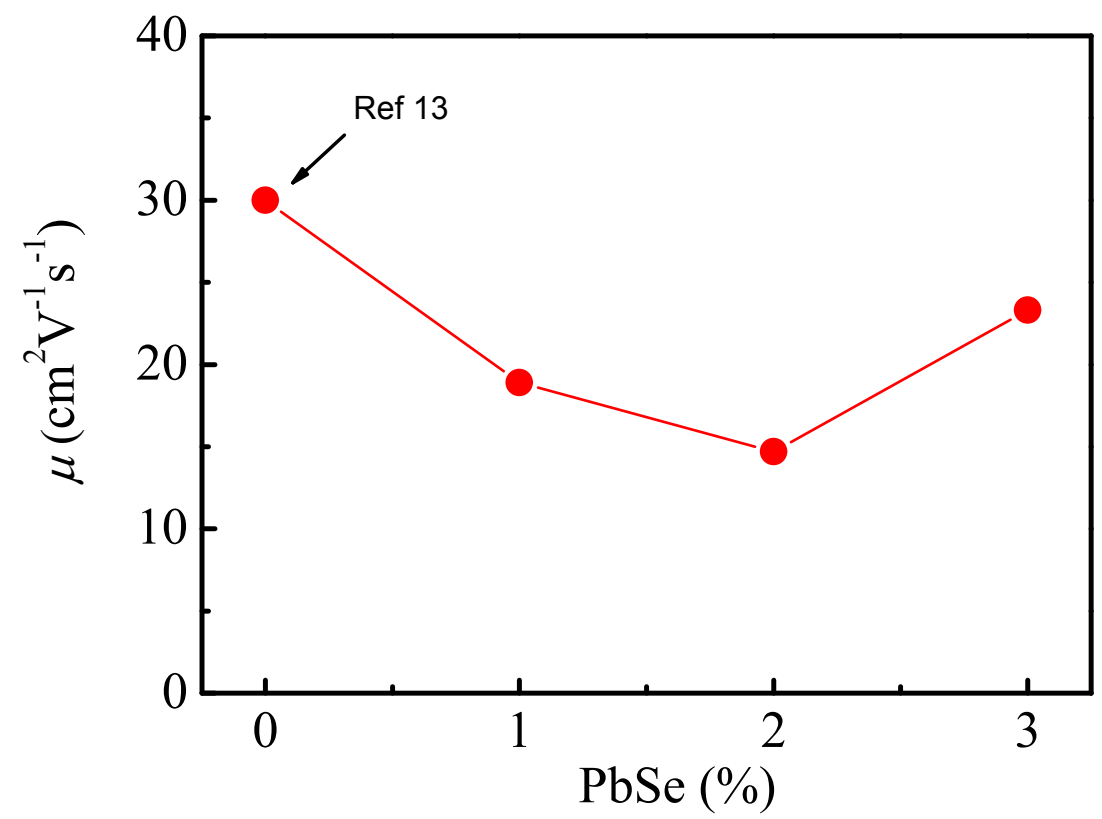


Figure S4. The reproducibility of thermoelectric properties as a function of temperature of $\mathrm{SnSe}+1 \% \mathrm{PbSe}$ measured along the pressing direction. (a) Electrical conductivity $(\sigma)$. (b) Seebeck coefficient $(S)$. (c) Thermal conductivity ( $\kappa)$. (d) ZT.
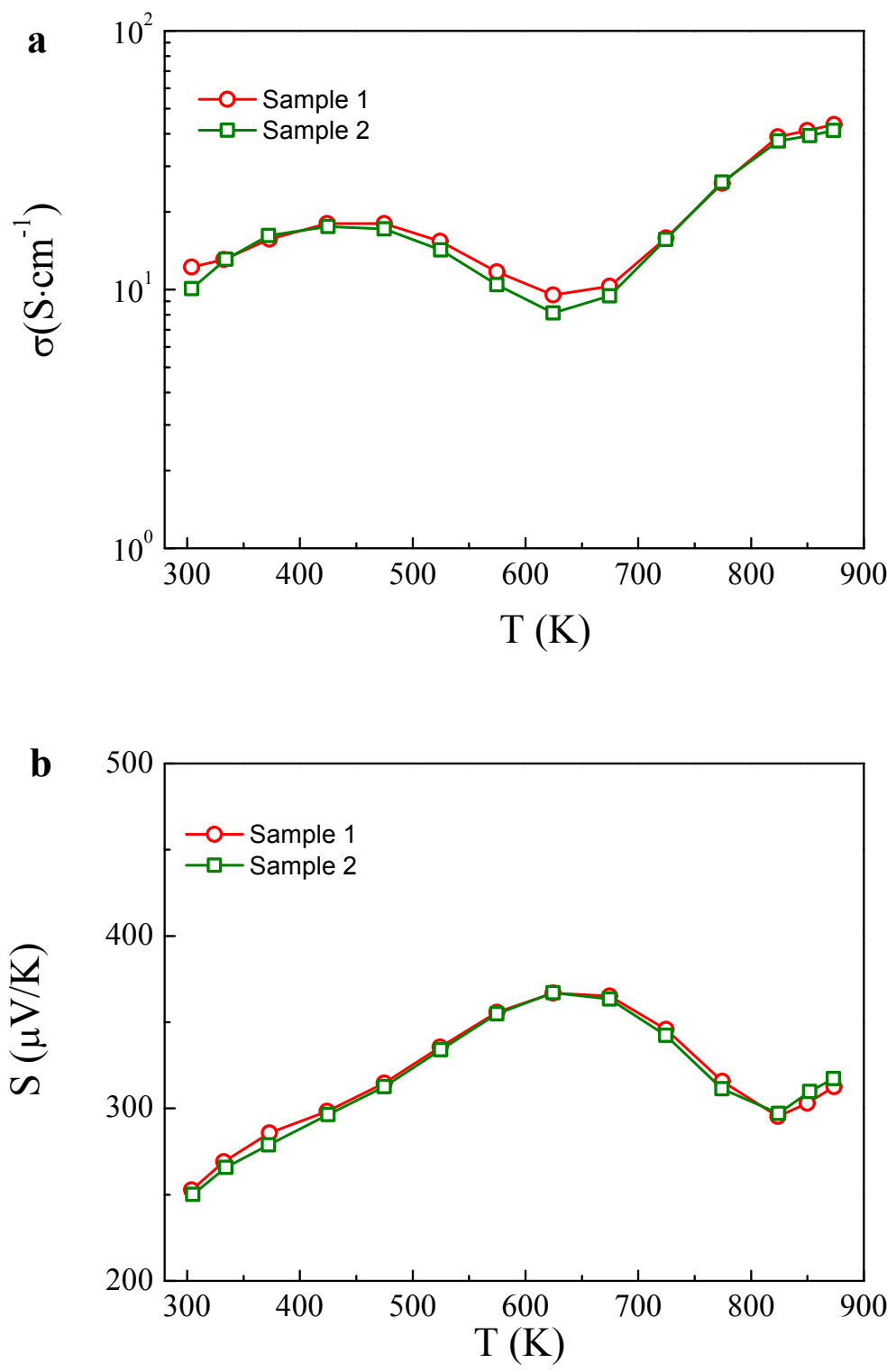

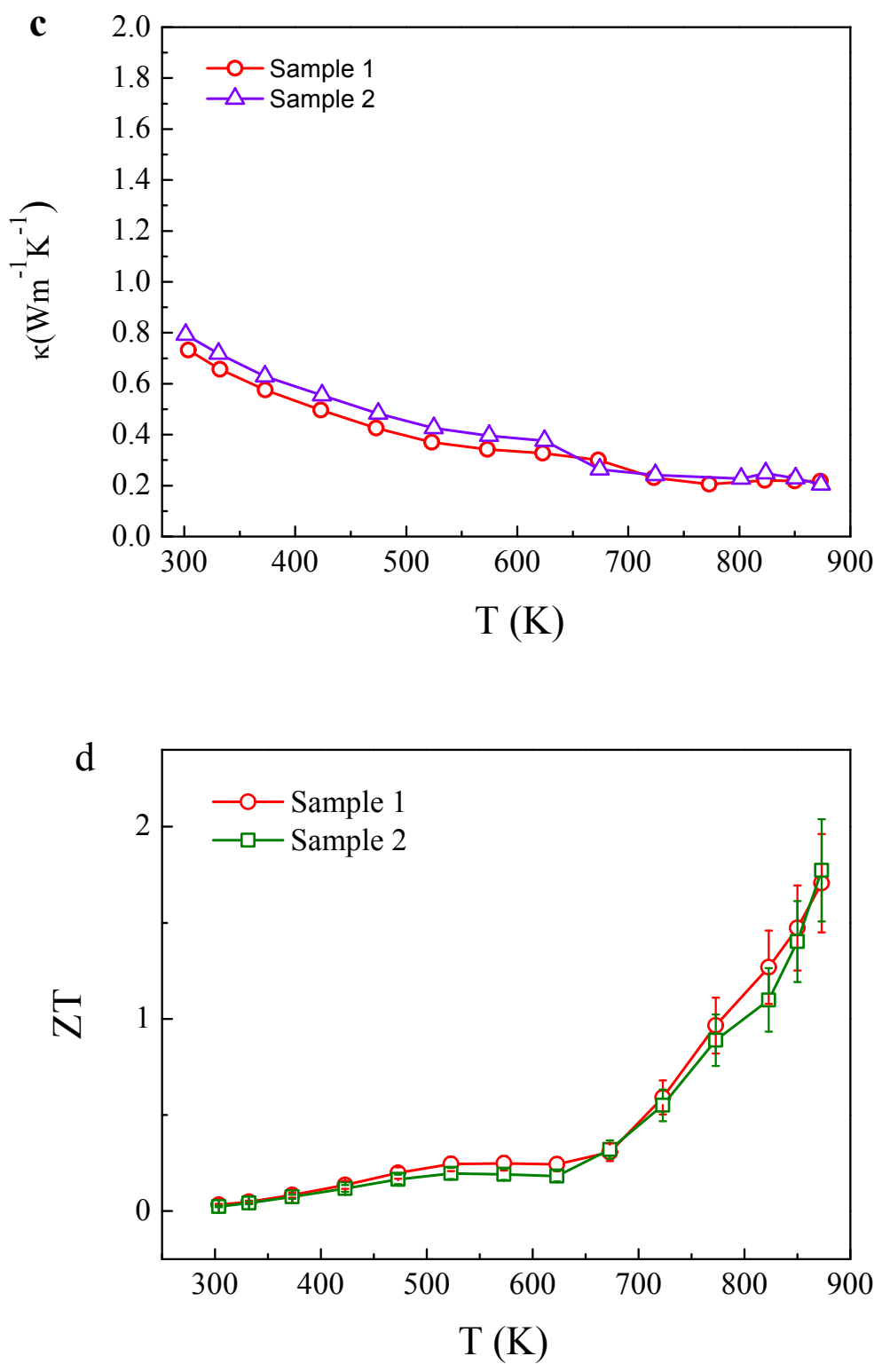
Figure S5. XRD pattern with Rietveld refinement for $\mathrm{Pb}$-free $\mathrm{SnSe}$ prepared by the same process. All the peaks can be indexed with the cubic and orthorhombic phase of SnSe. Below the experimental data fitted by a red line, there are two rows of vertical blue bars and a green line. The green line represents the difference between the experimental XRD data and our Rietveld refinement data. The upper blue bars represent the peak positions for Pnma phase of SnSe, while the lower blue bars for cubic phase SnSe.

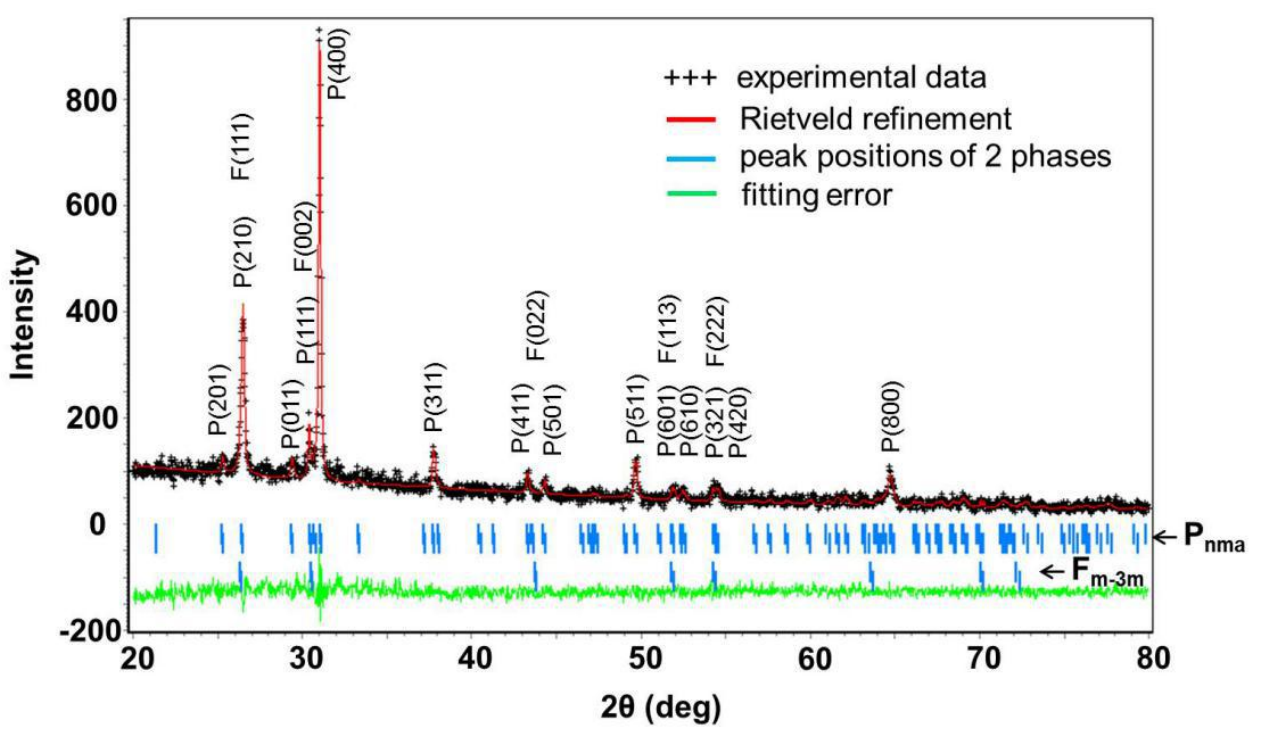

